\title{
Разработка программно-методического обеспечения гидродинамики и динамики подводных аппаратов ${ }^{1}$
}

Представлен предметно-ориентированный программно-методический комплекс, позволяющий эффективно выполнять комплексное моделирование гидродинамики и динамики подводных аппаратов, последовательно решать частные задачи с учётом конструктивных особенностей аппарата, условий обтекания, способов обеспечения выхода аппарата из пускового устройства и устойчивого движения его по траектории.

Ключевые слова: гидродинамика, динамика, математическое моделирование, программный комплекс.

Разработка программно-методического обеспечения для моделирования и анализа результатов расчёта гидродинамики и динамики подводных аппаратов связана с необходимостью решения таких задач, как совместное использование математических моделей, описывающих физически разнородные процессы, исключение дублирования исходных данных и уменьшение подготовительной работы, ведение банка данных выполненных решений.

На рис. 1 представлена обобщённая схема программного комплекса Win Gid, paзработанного ОАО «ГРЦ Макеева» для решения задач гидродинамики и динамики подводного старта. В этом комплексе с единых методологических позиций осуществляется математическое моделирование процессов гидродинамики и динамики движения аппарата в воде при вертикальном или горизонтальном выходе из пускового устройства, а также при входе аппарата в воду [1]. Комплекс позволяет моделировать различные способы снижения гидродинамических нагрузок при старте, такие как переход от режима сплошного обтекания к кавитационному, применение газоструйной завесы и гидродинамических устройств, оптимизация формы аппарата. С целью облегчения управлением программным комплексом он снабжен интерактивной оболочкой, реализующей диалоговый режим с пользователем, обеспечивающей редактирование исходных данных, управление проектами решаемых задач и предоставляющей пользователю возможность отображения результатов в графическом виде.

Для моделирования гидродинамики и динамики подводных аппаратов применяется модульный принцип расчёта массоцентровочных

\footnotetext{
Работа поддержана РФФИ. Грант 14-08-00128.
}

и гидродинамических характеристик, определения траекторий движения, вычисления действующих на аппарат механических нагрузок. Структура вычислений частных задач строится по принципу «вход-выход», и результаты решения одной частной задачи являются исходными данными для последующих задач. Такой подход позволяет автоматизировать обмен данными между частными задачами, исключить дублирование и итерационные процедуры.

В качестве исходных данных для расчёта массоцентровочных характеристик используются заданные распределения масс конструкции, сосредоточенные массы конструкции и распределения массы топлива.

Задача определения гидродинамических характеристик аппарата является базовой, на основе решения которой строятся задачи динамики и определения механических нагрузок аппарата. В ней рассматривается тело вращения, состоящее из цилиндрического корпуса и притупленной носовой части. Силы потенциального и вязкого происхождения рассчитываются независимо друг от друга. Результаты расчёта используются на всех последующих этапах моделирования [2, 3].

Задача динамики аппарата представлена двумя независимыми задачами: определение параметров продольного движения в пусковом устройстве и расчёт параметров углового движения в пусковом устройстве, в воде и при пересечении поверхности воды. Разделение общей задачи динамики при движении аппарата в пусковом устройстве на задачи определения продольного и углового движения связано как с предположением о малом взаимном влиянии этих составляющих движения друг на друга, 


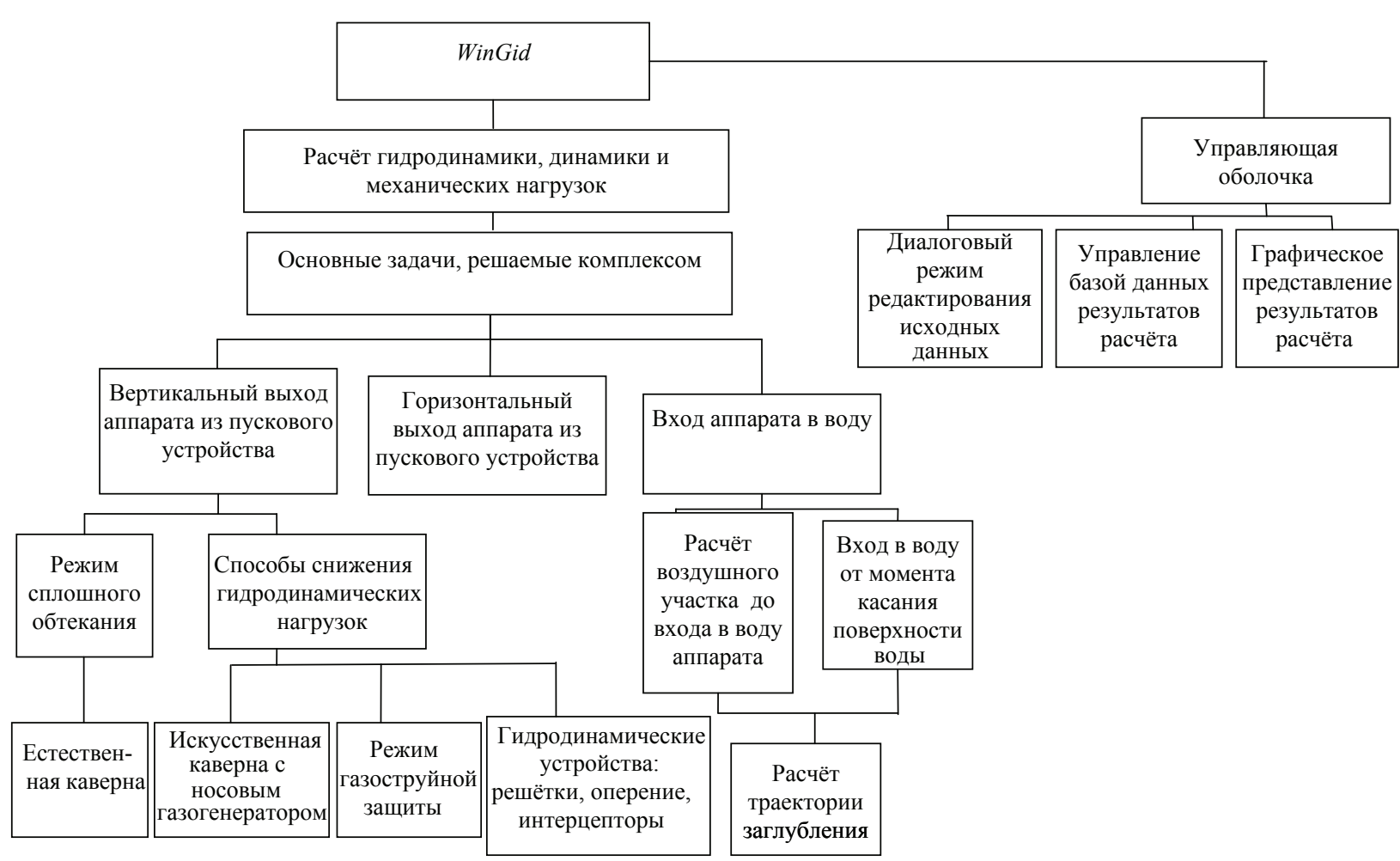

Рис. 1. Обобщенная схема функциональных возможностей комплекса Win Gid

так и с необходимостью моделирования физически разнородных процессов. Так, в основе расчёта динамики продольного движения лежит моделирование газодинамических процессов, происходящих в свободных объёмах между пусковым устройством и аппаратом, а расчёта углового движения в пусковом устройстве - действие внешних сил, среди которых основное значение имеют силы гидродинамической природы и реакций амортизации.

Одной из главных проблем при расчёте газодинамических параметров в характерных объёмах является корректный учёт энергетических потерь, связанных с теплообменом между продуктами сгорания топлива, стенками пускового устройства и поверхностью самого аппарата. Энергетические потери в пусковом устройстве при пуске аппарата могут достигать 20-30 \% от общей энергии, поступающей от источников продуктов сгорания. Учёт влияния теплообмена проводится путём введения коэффициента, учитывающего теплопотери при вычислении температуры газов, поступающих в свободные объёмы.

Реализация математической модели, основные особенности которой определяются способом пуска, конструктивными особенно- стями пускового устройства и самого аппарата, позволяет:

решать проектные задачи выбора энергорасходной характеристики средства пуска;

рассчитывать тягу двигательной установки с учётом противодавления;

выполнять расчёт газовой полости, формирующейся в районе среза пускового устройства совместно с процессом формирования искусственной каверны аппарата;

рассчитывать динамику развития струйной газовой защиты;

рассчитывать гидродинамические характеристики аппарата с учётом текущих параметров динамики.

Результатом решения задачи являются определение параметров продольного движения аппарата, величины давлений в характерных объёмах и уточнёние гидродинамических характеристик.

После определения параметров продольного движения аппарата в пусковом устройстве решается задача динамики углового движения, математическая модель которой строится на базе уравнений Эйлера - Лагранжа. Это позволяет выполнить расчёт перемещений аппарата относительно удароопасных сечений пусково- 
го устройства с учётом движения подводной платформы, волнения моря, конструктивных особенностей пускового устройства. Угловое движение аппарата в пусковом устройстве происходит под действием сил, обусловленных взаимодействием аппарата и воды, а также реакций системы амортизации между пусковым устройством и аппаратом. Программный комплекс позволяет выполнять оценку различных способов снижения уровня гидродинамических нагрузок, таких как формирование искусственной каверны или струйной газовой завесы, которые улучшают гидродинамические характеристики аппарата, рассчитанные с учётом двухфазного обтекания и размеров газовой полости. Если рассматриваемый аппарат характеризуется значительным удлинением, то для него может производиться расчёт динамики углового движения с учётом упругих деформаций корпуса. Учёт упругих деформаций осуществляется с использованием балочного представления корпуса аппарата. Для определения гидродинамических нагрузок проводится расчёт коэффициентов инерционных, позиционных и вращательных сил. Они описывают нестационарное взаимодействие упругого аппарата с жидкостью, учитывают такие кинематические параметры, как угол атаки, угловая скорость, обобщённые координаты и обобщённые скорости. Система дифференциальных уравнений, описывающих динамику аппарата, в этом случае расширяется, и совместно с системой уравнений Эйлера - Лагранжа решается система уравнений Лагранжа, описывающая упругие деформации корпуса аппарата.

Эта же математическая модель используется и при расчёте параметров динамики аппарата при движении на подводном участке траектории, пересечении воды и начальном воздушном участке или при входе в воду. Отличие состоит лишь в том, что в уравнениях отсутствуют силы реакций опорно-ведущих поясов. Математическая модель также позволяет учитывать такие особенности, как расчёт гидродинамических коэффициентов при наличии интерцепторов, решётчатых рулей или хвостового оперения. Существует возможность расчёта тяги двигательной установки аппарата с учётом противодавления, что позволит решать задачи управления.

Важной задачей является определение механических нагрузок, действующих на аппарат. Для их вычисления вводится локальная система координат, например, от носка до рассматриваемого сечения аппарата. Для этой системы применяется принцип Даламбера: при движении в каждый момент внешняя сила, внутренняя сила и сила инерции в рассматриваемом сечении аппарата, а также моменты этих сил относительно какого-либо центра взаимно уравновешиваются. Использование этого принципа позволяет строить распределения внутренних сил и моментов, действующих в конструкции аппарата по длине. Задача решается совместно с задачами динамики.

На рис. 2 представлен вид рабочего окна программного комплекса Win Gid. В левой части окна расположены элементы для управления проектами, отображения состава проекта и хода его решения, в правой - элементы, отображающие результаты расчёта как в табличном, так и в графическом виде.

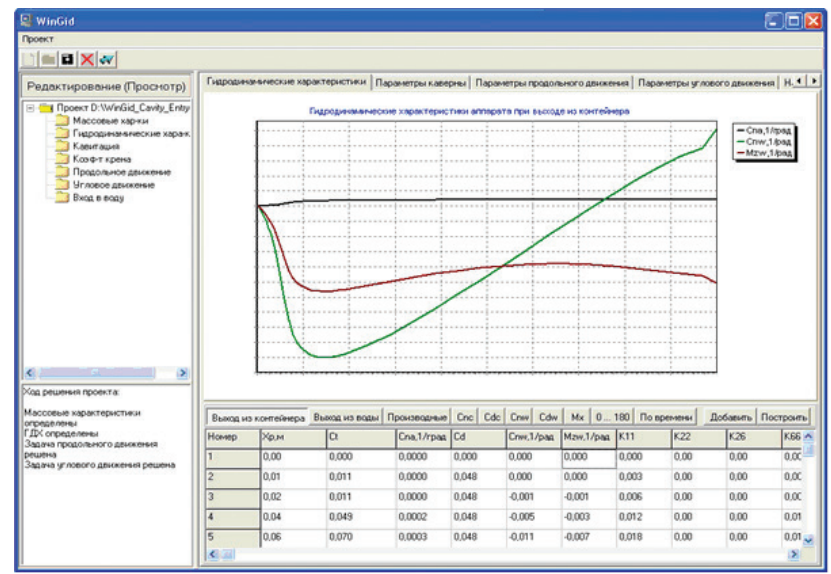

Рис. 2. Рабочее окно программного комплекса Win Gid

Корректность расчётов, выполненных с помощью программного комплекса, подтверждается модельными экспериментами, такими как вход аппарата в воду и его последующее погружение, вертикальный выход аппарата из пускового устройства с последующим движением в воде и выходом из воды, что позволяет продемонстрировать основные возможности программного комплекса.

Сравнение последовательных расчётных 


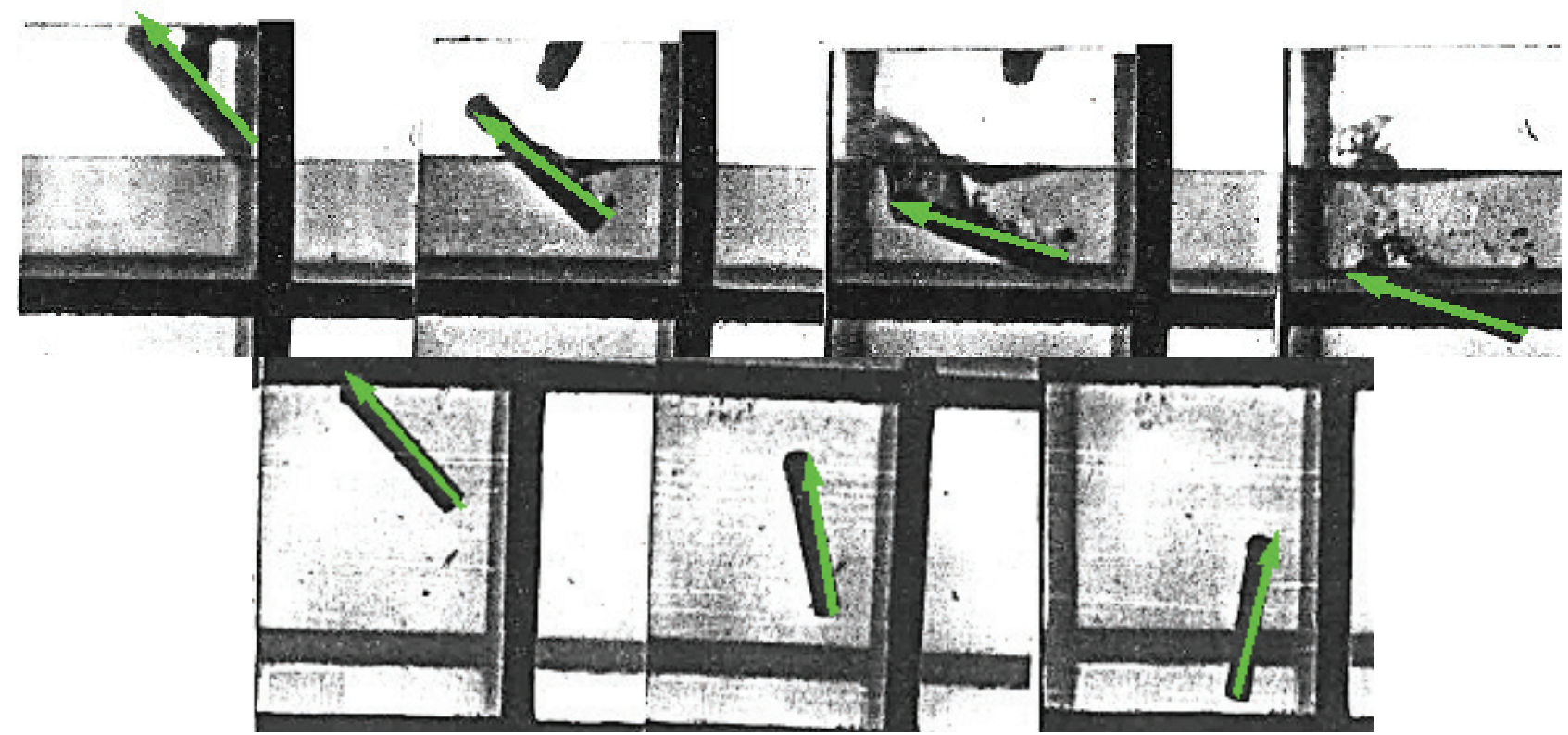

Рис. 3. Сравнение расчётных и экспериментальных положений при входе в воду и погружений в гидробассейне. Вектор указывает расчётное положение аппарата

и экспериментальных положений аппарата (рис. 3) показывает достаточно хорошую сходимость результатов, что подтверждает достоверность используемой математической модели, которая отражает основные особенности сопровождающих физических процессов: движение в естественной нестационарной каверне, исчезающей с увеличением глубины погружения, движение аппарата в широком диапазоне углов атаки.

Сравнение расчётных и экспериментальных параметров, описывающих динамику аппарата в пусковой установке, при движении в воде и при выходе из воды, приведены на рис. 4-6.

На графиках используются следующие обозначения: $V_{x}, V_{y}$ - проекции линейной скорости центра масс аппарата; $V_{x}^{\max }, V_{y}^{\max }-$ мак-

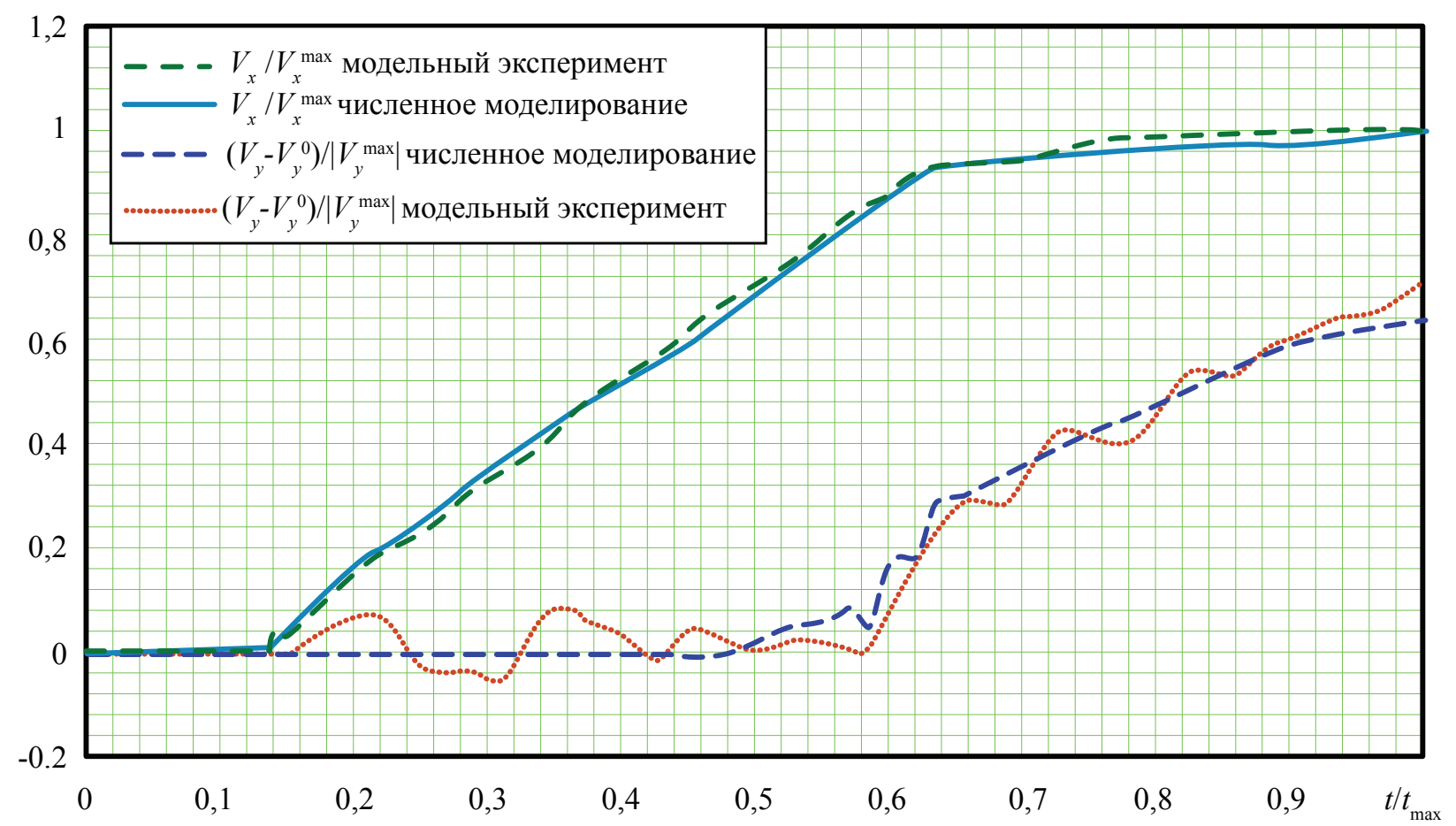

Рис. 4. Сравнение расчётных и экспериментальных проекций линейных скоростей центра масс аппарата 


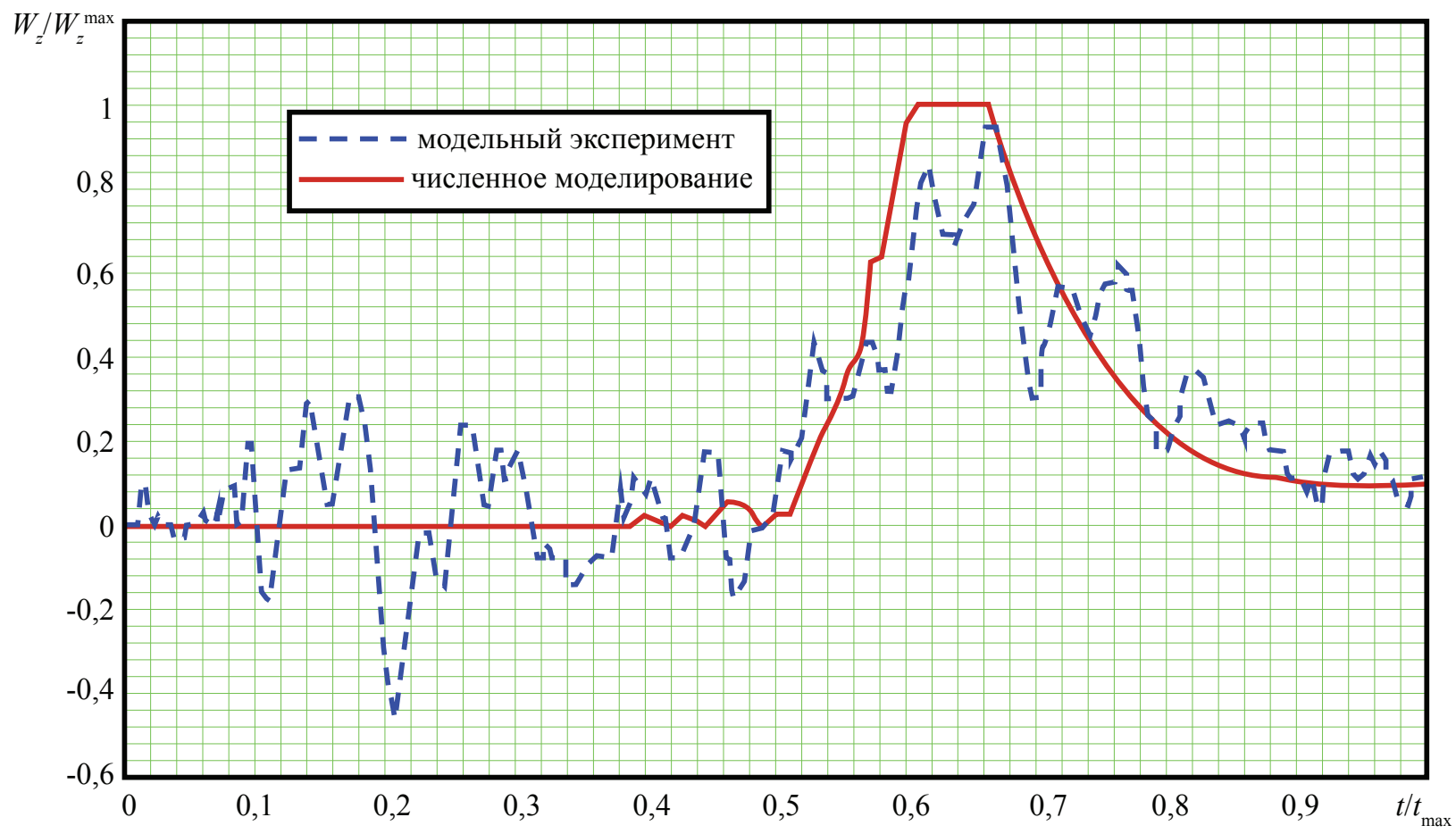

Рис. 5. Сравнение расчётной и экспериментальной проекций угловой скорости аппарата

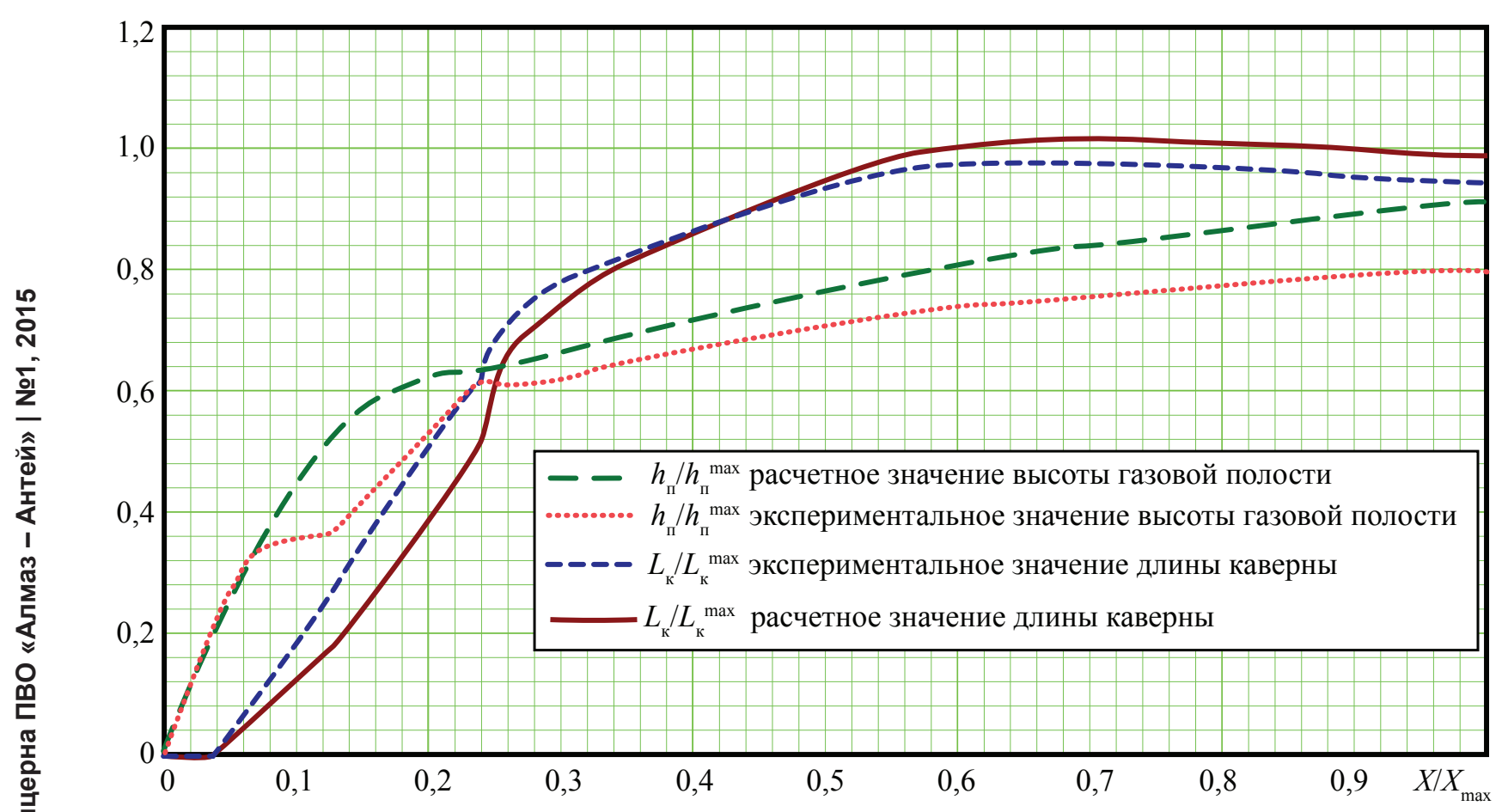

Рис. 6. Сравнение расчётных и экспериментальных параметров каверны и газовой полости

симальные значения проекций скорости центра масс; $V_{y}^{0}-$ проекция линейной скорости платформы; $\left|V_{y}^{\max }\right|$ - модуль проекции макси$\stackrel{\curvearrowright}{\mp}$ мальной линейной скорости аппарата; $W_{z}, W_{z}^{\max }$ - проекция угловой скорости и её максимальное значение; $t, t$ max - текущее время и полное время движения; $L_{\mathrm{\kappa}}, L_{\mathrm{\kappa}}^{\max }-$ длина каверны и её $\underline{\underline{0}}$ максимальное значение; $h_{\pi}, h_{\Pi}^{\max }-$ высота газо- вой полости над пусковым устройством и её максимальное значение; $X, X^{\max }$ - вертикальный путь аппарата и его максимальное значение. Получено удовлетворительное соответствие расчётных и экспериментальных параметров.

Приведённые примеры показывают эффективность разработанного комплекса WinGid, который находит широкое примене- 
ние в инженерных расчётах.

\section{Список литературы}

1. Дегтярь В. Г., Пегов В. И. Гидродинамика подводного старта ракет. М.: Машиностроение, 2009. $448 \mathrm{c.}$

2. Дегтярь В. Г., Пегов В. И. Гидродинамика баллистических ракет подводных лодок. Ми- acc: ФГУП «ГРЦ КБ им. академика В. П. Макеева», 2004. 256 с.

3. Дегтярь В. Г., Пегов В. И. Математические модели гидродинамики ракет // Математическое моделирование: сб. науч.-метод. тр. Челябинск: ЮУрГУ, 2003. С. 13-43.

\section{Поступила 30.07.14}

Дегтярь Владимир Григорьевич - доктор технических наук, профессор, член-корреспондент РАН, академик РА$\mathrm{PAH}$, генеральный директор - генеральный конструктор ОАО «Государственный ракетный центр имени академика В. П. Макеева», г. Миасс Челябинской обл.

Область научных интересов: создание баллистических ракет подводных лодок, межконтинентальных баллистических ракет, ракетно-космических комплексов, системное проектирование, прикладная гидродинамика и аэродинамика, механика конструкций из композиционных материалов, материаловедение.

Пегов Валентин Иванович - доктор технических наук, профессор, ведущий научный сотрудник отдела фундаментальных проблем аэрокосмических технологий Челябинского научного центра УрО РАН, г. Миасс Челябинской обл. Область научных интересов: гидродинамика, газодинамика, динамика летательных аппаратов.

Мошкин Игорь Юрьевич - кандидат технических наук, младший научный сотрудник отдела фундаментальных проблем аэрокосмических технологий Челябинского научного центра УрО РАН, г. Миасс Челябинской обл.

Область научных интересов: гидродинамика, тепломассообмен.

Степанов Владимир Викторович - ведущий математик ОАО «Государственный ракетный центр имени академика В. П. Макеева», г. Миасс Челябинской обл.

Область научных интересов: динамика полёта летательных аппаратов, гидродинамика.

Семёнов Андрей Александрович - начальник группы ОАО «Государственный ракетный центр имени академика В. П. Макеева», г. Миасс Челябинской обл.

Область научных интересов: динамика полёта летательных аппаратов, газодинамика, гидродинамика, тепломассообмен.

Хлыбов Владимир Ильич - доктор физико-математических наук, начальник отдела ОАО «Государственный ракетный центр имени академика В. П. Макеева», г. Миасс Челябинской обл.

Область научных интересов: аэродинамика, гидродинамика, тепломассообмен. 